\title{
Questes
}

\section{Copie, authenticité, originalité : éléments}

bibliographiques

Jean-Baptiste Camps, Magali Cheynet et Vincent Le Quentrec

\section{(2) OpenEdition}

Édition électronique

URL : http://journals.openedition.org/questes/3594

DOI : 10.4000/questes.3594

ISSN : 2109-9472

Éditeur

Les Amis de Questes

\section{Édition imprimée}

Date de publication : 15 janvier 2015

Pagination : 159-168

ISSN : 2102-7188

\section{Référence électronique}

Jean-Baptiste Camps, Magali Cheynet et Vincent Le Quentrec, « Copie, authenticité, originalité :

éléments bibliographiques », Questes [En ligne], 29 | 2015, mis en ligne le 24 juin 2015, consulté le 15 septembre 2020. URL : http://journals.openedition.org/questes/3594

\section{(C) Association des amis de «Questes »}




\section{Éléments bibliographiques}

\section{Bibliographie « diplomatique »}

\section{Édition des textes documentaires}

Conseils pour l'édition des textes médiévaux : fascicule II, Actes et documents d'archives, dir. Olivier GuYotJEAnNIn, Paris, Comité des travaux historiques et scientifiques et École nationale des chartes, coll. « Orientations et méthodes », $2001^{1}$ et $2009^{2}$.

\section{Diplomatique}

Gli Atti privati nel tardo medio evo : fonti per la storia sociale, dir. Paolo BREZzI et Egmont LEE, Roma/Toronto, Istituto di studi romani, coll. « Atti di studi romani », 1984.

Les Cartulaires, Actes de la Table ronde (5-7 décembre 1991, Paris), Paris, École des Chartes/Champion, coll. «Mémoires et documents de l'École des chartes », 1993, p. 91-104.

La Diplomatique française $d u$ haut Moyen Âge: inventaire des chartes originales antérieures à 1121 conservées en France, tome 1, dir. Michèle CourTOIs, Turnhout, Brepols, 2001.

Guyotjeannin, Olivier, Pycke, Jacques, et Tock, Benoît-Michel, Diplomatique médiévale, Turnhout, Brepols, coll. « L'atelier du médiéviste », 2, 2006.

Notaires et crédit dans l'Occident méditerranéen médiéval, dir. François MENANT et Odile REDON, Rome, École française de Rome, 2004.

Notariado publico y documento privado: de los origenes al siglo xiv, actas del VII Congreso internacional de diplomatica, dir. José TRENCHS ODENA, Valencia, Conselleria de cultura, educacio I ciencia, 1989, 2 vol.

Vocabulaire international de la diplomatique [1994], dir. Maria MiLAGROS et Cárcel ORTí, Valencia, Universidad de València, 1997 ( $2^{\mathrm{e}}$ édition).

\section{Divers}

Dictionnaire de la culture juridique, dir. Denis Alland et Stéphane Rials, Paris, Lamy, coll. « Quadrige, dicos de poche », 2003.

\section{Théories et pratiques de l'édition des textes médiévaux et leur histoire.}

\section{Bibliographies et généralités}

Nous renvoyons, pour la partie relative aux différentes théories et pratiques dans le domaine de l'édition des textes, aux bibliographies proposées par : 
Bourgain, Pascale et Vielliard, Françoise, Conseils pour l'édition des textes médiévaux: fascicule III, Textes littéraires, Paris, Comité des travaux historiques et scientifiques \& École nationale des chartes, coll. « Orientations et méthodes », 2002.

Foulet, Alfred et SpeER, Mary Blakely, On editing Old French texts, dir., Lawrence, The Regents Press of Kansas, 1979.

Huygens, Robert Burchard Constantijn, Ars edendi : introduction pratique à l'édition des textes latins du Moyen Âge, Turnhout, Brepols, 2001.

Pratiques philologiques en Europe : actes de la journée d'étude organisée à l'École des Chartes le 23 septembre 2005, dir. Frédéric Duval, Paris, École des Chartes, coll. « Études et rencontres de l'École des Chartes », 21, 2006.

Probable Truth: Editing Medieval Texts from Britain in the Twenty-First Century, dir. Vincent Gillespie et Anne Hudson, Turnhout, Brepols, coll. "Texts and Transitions », 5, 2013.

Rochebouet, Anne, "Les éditions de texte en ancien français : bibliographie sélective », Reverdie, 2007, en ligne: http://reverdie.free.fr/site/IMG/pdf/ Bibliographieeditions_texte.pdf (page consultée le 21 décembre 2014).

\section{Lachmann et la méthode généalogique}

Fiesol, Giovanni, La Genesi del Lachmannismo, Firenze, Sismel Edizioni del Galluzzo, coll. « Millennio Medievale », 2000.

FORNARO, Sotera, « Karl Lachmann et sa méthode », Revue germanique internationale, 14, 2011, p. 125-138, URL : http://rgi.revues.org/1281, consulté le 6 janvier 2015.

MAAS (Paul), «Leitfehler und stemmatische Typen », Byzantinische Zeitschrift, 37, 2, 1937, p. 289-294.

—, Textkritik [1927], $4^{\mathrm{e}}$ édition, Leipzig, B. G. Teubner, 1960.

PARIS, Gaston, et PANNIER, Léopold, La Vie de saint Alexis : poème du XI siècle et renouvellements des XII $I^{e}$ XIII et $X I V^{e}$ siècles, Paris, A. Franck, coll. « Bibliothèque de l'École des hautes études », 1872.

PASQuAl, Giorgio, Storia della tradizione e critica del testo [1934], Firenze, Le Lettere, $1988,2^{\mathrm{e}}$ édition.

Timpanaro, Sebastiano, La Genesi del metodo del Lachmann [1963], $4^{\mathrm{e}}$ éd., Torino, UTET Libreria, 2003.

Trovato, Paolo, Everything you always wanted to know about Lachmann's method: a non-standard handbook of genealogical textual criticism in the age of poststructuralism, cladistics, and copy-text, Limena, Libreriauniversitaria.it, 2014.

\section{L'intervention de Joseph Bédier et les débats entre « lachmanniens » et « bédiéristes »}

BÉDIER, Joseph, Joseph Bédier, « Du classement des manuscrits », dans Jean Renart, Le lai de l'ombre, éd. J. Bédier, Paris, Société des anciens textes français, 1913, p. XXIIIXLV. 
—, « La tradition manuscrite du Lai de l'ombre : réflexions sur l'art d'éditer les anciens textes », Romania, 54, 1928, p. 161-196 et 321-356.

Castellani, Arrigo, Bédier avait-il raison? La méthode de Lachmann dans les éditions de textes $d u$ Moyen Âge, Fribourg, Éditions universitaires, coll. «Discours universitaires », 1957.

Collomp, Paul, La Critique des textes, Strasbourg/Paris, Publications de la Faculté des Lettres de l'Université de Strasbourg/Société d'Édition des Belles Lettres, coll. « Initiation, méthode », 1931.

Monfrin, Jacques, «Problèmes d'édition de texte», dans Critique et édition de textes: actes du XVII congrès international de linguistique et philologie romanes (Aix-enProvence, 29 août-3 septembre 1983), Aix-en-Provence, Université de Provence, 1986, p. 353-364.

RoQUES, Gilles, «L'édition des textes français entre les deux guerres », dans Histoire de la langue française, 1914-1945, dir. Gérald ANTOINE et Robert MARTIN, Paris, CNRS, 1995, p. 993-1000.

—, "Les éditions de texte », dans Histoire de la langue française, 1945-2000, dir. Gérald ANTOINE, Bernard CERQUIGLINI, Paris, CNRS, 2000, p. 867-882.

- Pour le débat à Naples en 1974 entre Roncaglia, Segre et Lecoy, voir :

Atti del XIV Congresso Internazionale di Linguistica e Filologia Romanza (Napoli, 15-20 aprile 1974), dir. Alberto VÁRVARO, Naples/Amsterdam, G. Macchiaroli/ J. Benjamins, vol. 1: "Sedute plenarie e tavole rotonde», et notamment: "Tavola rotonda: Premesse ideologiche della critica testuale» (p. 479-514), ainsi que les interventions d'Aurelio Roncaglia, «La critica testuale» (p. 481-488), Duncan McMillan, «Critique textuelle : principes et méthodes, p. 489-492, Cesare SEGRE, «La critica testuale » (p. 493-500) et Félix LECOY, «L'édition critique des textes » (p. 501-508), ainsi que la discussion (p. 509-514).

\section{Philologie et manuscrits}

ANTONELLI, Roberto, «Interpretazione e critica del testo », dans Letteratura italiana, IV, L'Interpretazione, dir. Alberto AsOR RosA, Torino, Giulio Einaudi, 1985, p. 141-243.

Avalle, D'Arco Silvio, La Letteratura medievale in lingua d'oc nella sua tradizione manoscritta : problemi di critica testuale, Torino, Giulio Einaudi, 1961.

-, Principi di critica testuale, Padova, Antenore, 1972.

-, La Doppia Verità : fenomenologia ecdotica e lingua letteraria nel Medioevo romanzo, Tavarnuzze/Firenze, SISMEL,Edizioni del Galluzzo/Fondazione Ezio Franceschini, coll. « Archivio romanzo », 2002.

BusBy, Keith, Codex and context : reading old French verse narrative in manuscript, Amsterdam/New York, Rodopi, coll. « Faux titre », 2 vol., 2002.

CAReri, Maria, Hasenohr, Geneviève, FÉry-Hue, Françoise, Gasparri, Françoise, LABOry, Gilette, LefÈvre, Sylvie, Leurquin, Anne-Françoise et RubY, Christine, Album de manuscrits français du XIIIe siècle, Rome, Viella, 2001. 
-, Saint-Pol Ruby, Christine de, Short, Ian, Nixon, Terry et Stirnemann, Patricia, Livres et écritures en français et en occitan au XII siècle : catalogue illustré, Rome, Viella, coll. « Scritture e libri del Medioevo », 2011.

DAIN, Alphonse, Les Manuscrits, Paris, Les Belles lettres, coll. «Collection d'études anciennes », 1949.

La Filologia romanza e i codici, Atti del convegno, Messina, 19-22 dicembre 1991, dir. Saverio Guida et Fortunata LATElla, Messina, Sicania, 2 vol., 1993.

FrANDSEN, Stig Ramlev, « La dialectique de la variance. Nouvelle philologie et stratégies interprétatives du texte médiéval ", Cahiers de civilisation médiévale, t. 48, 2005, p. 111-127, en ligne: http://www.persee.fr/web/revues/home/prescript/article/ccmed 0007-9731_2005_num_48_190_2903 (page consultée le 15 décembre 2014).

GRÖBER, Gustav, «Die Liedersammlungen der Trobadours », Romanische Studien, 2, 1877, p. 337-670.

« Intavulare ». Tables de chansonniers romans, 1998, série divisée en trois sous-séries, «I. Canzonieri provenzali », dir. Anna Ferrari, « II. Chansonniers français », dir. Madeleine Tyssens et « III. Canzonieri italiani », dir. Giovanni Borriero.

Lyrique romane médiévale : la tradition des chansonniers. Actes du colloque de Liège, 1989, dir. Madeleine TYSSENS, Liège, Université de Liège, coll. «Bibliothèque de la Faculté de Philosophie et de Lettres de l'Université de Liège », 1991.

Les Manuscrits de Chrétien de Troyes, dir. Keith Busby, Terry NixOn, Alison Stones (et al.), Amsterdam, Rodopi, coll. « Faux titre », 2 vol., 1993.

Mise en page et mise en texte du livre manuscrit, dir. Henri-Jean MARTIN et Jean VEZIN, Paris, Éditions du Cercle de la Librairie-Promodis, 1990.

Moderna, 10-2, 2008, La Materialità nella filologia, dir. Alberto CADIOLI et Maria Luisa MENEGHETTI; voir particulièrement Federico SAVIOTTI, «Filologia materiale, Philologie matérielle, Material philology», p. 147-151, et la bibliographie, «Repertorio Bibliografico ragionato: la materialità nella filologia (1985-2007)», p. 157-225.

Schwan, Eduard, Die Altfranzösischen Liederhandschriften, ihr Verhältniss, ihre Entstehung und ihre Bestimmung. Eine litterarhistorische Untersuchung, Berlin, Weidmann, 1886. Ils ont remplacé ceux de Gaston RAYNAUD, Bibliographie des chansonniers français des XIII et XIV ${ }^{e}$ siècles, Paris, F. Vieweg, 1884, 2 tomes.

SEgRe, Cesare, "Critique textuelle, théorie des ensembles et diasystème », Bulletin de la classe des lettres et des sciences morales et politiques de l'Académie royale de Belgique, 62, 1976, p. 279-292.

-, "Les transcriptions en tant que diasystèmes », dans La Pratique des ordinateurs dans la critique des textes, dir. Jean Glenisson, Jean Irigoin, Robert Marichal (et al.), Paris, Éditions du Centre national de la recherche scientifique, 1979, p. 45-49.

ZINELLI, Fabio, « Gustav Gröber et les manuscrits de troubadours », dans Le Moyen Âge au miroir du XIX siècle (1850-1900), dir. Laura KENDRICK, Francine MORA-LEBRUN et Martine REID, 2003, p. 119-145. 


\section{$L^{\prime}$ « Éloge de la variante » et la « nouvelle philologie »}

Alte und neue Philologie, dir. Martin-Dietrich GleßGEN et Franz LeBSAnft, Tübingen, M. Niemeyer, coll. « Beihefte zu Editio », 1997.

Cerquiglini, Bernard, Éloge de la variante. Histoire critique de la philologie, Paris, Éditions du Seuil, coll. « Des travaux », 1989.

FrandSEN, Stig Ramlev, «La dialectique de la variance. Nouvelle philologie et stratégies interprétatives du texte médiéval », Cahiers de civilisation médiévale, t. 48, ${ }^{\circ} 190$, 2005, p. 111-127.

Speculum, 65/1, 1990, The New Philology; voir notamment Stephen G. Nichols, «Introduction : Philology in a Manuscript Culture », p. 1-10.

Towards a Synthesis? Essays in the New Philology, dir. Keith BusBY, Amsterdam/Atlanta, coll. «Faux titre », 1993 ; notamment Keith BusBY, "Variance and the Politics of Textual Criticism », p. 29-45.

Tyssens, Madeleine, "Philologie "chevronnée", nouvelle philologie», Revue de linguistique romane, 2002, 66, p. 403-420.

\section{La « philologie numérique »}

ANDREwS, Tara, «The third way: philology and critical edition for a digital age ", Variants: The Journal of the European Society for Textual Scholarship, 2012, 10, p. 61-76, en ligne, URL: https://lirias.kuleuven.be/bitstream/123456789/352304/2/ variants_postprint.pdf (page consultée le 20 décembre 2014).

Electronic Publishing: Politics and Pragmatics, dir. Gabriel Egan, Tempe, Arizona Center for Medieval and Renaissance Studies, coll. «Medieval and Renaissance Text Studies », 2010.

Electronic Texts : investigations in the method and theory, dir. Kathryn SuTHERLAND, Oxford, Clarendon Press, 1997.

FISCHER, Franz, «All texts are equal, but... Textual Plurality and the Critical Text in Digital Scholarly Editions », Variants : The Journal of the European Society for Textual Scholarship, 2012, 10, p. 77-92, en ligne, URL : http://kups.ub.uni-koeln.de/5056/ (page consultée le 20 décembre 2014).

La Pratique des ordinateurs dans la critique des textes, éd. Robert MARICHAL, Jean GLENISSON, Jean IRGOIN et Jacques MONFRIN, Paris, 1979.

Probable Truth: editing medieval texts from Britain in the twenty-first century, dir. Vincent GILlesPIE et Anne Hudson, Turnhout, Brepols, coll. « Texts and transitions », 2013.

RoBINSON, Peter, « Towards a Theory of Digital Editions », Variants : The Journal of the European Society for Textual Scholarship, 2012, 10, p. 105-131, en ligne, URL : http://www.academia.edu/3233227/Towards_a_Theory_of_Digital_Editions (page consultée le 20 décembre 2014)

TEI Consortium, TEI P5: Guidelines for Electronic Text Encoding and Interchange, 2014, URL: http://www.tei-c.org/release/doc/tei-p5-doc/en/html/ (consulté en novembre 2014. 


\section{Étude des processus de copie, critique textuelle, stemmatologie}

Alessio, Gian Carlo, «Edizioni medievali », dans Lo Spazio letterario del Medioevo, 1. Il Medioevo latino, III, La Ricezione del testo, dir. Guglielmo CAVAllo, Rome, Salerno, 1995, p. 29-58.

Bourgain, Pascale, "Sur l'édition des textes littéraires latins médiévaux », dans Bibliothèque de l'École des Chartes [en ligne], t. 150, 1992, 1, p. 5-49, URL : http://www.persee.fr/web/revues/home/prescript/article/bec_0373-6237_1992_num_ 150_1_450642 (page consultée le 9 janvier 2011).

Canettieri, Paolo, Loreto, Vittorio, Rovetta, Marta, et SAntini, Giovanna, « Philology and Information Theory ", Cognitive Philology [en ligne], 2009, 1, URL: http://ojs. uniroma1.it/index.php/cogphil/article/view/8816 (page consultée le 14 décembre 2014).

DeEs, Anthonij, «Vers une philologie mieux définie », Le Moyen français, n 39/40/41, Autour de Jacques Monfrin. Néologie et création verbale [en ligne], dir. Giuseppe DI Stefano et Rose M. BIDLER, Montréal, Éditions CERES, 1996-1997, p. 173-181, URL : http://cat.inist.fr/?aModele=afficheN\&cpsidt=2019315 (page consultée le 18 février 2012).

The Evolution of Texts : Confronting Stemmatological and Genetical Methods, Proceedings of the International Workshop Held in Louvain-la Neuve on September 12, 2004, dir. Caroline MAcÉ, Philippe V. BARET, Andrea Bozzi et Laura Cignoni, Pisa/Roma, Istituti editoriali e poligrafici internationali, 2006, publié dans Linguistica computazionale, 24-25, 2004/2005.

Froger, Dom Jacques, La Critique des textes et son automatisation, Paris, Dunod, coll. « Initiation aux nouveautés de la science », 1968.

Havet, Louis, Manuel de critique verbale appliquée aux textes latins, Paris, Hachette, 1911.

MARICHAL, Robert, « La critique des textes », dans L'Histoire et ses méthodes, dir. Charles SAMARAN, Paris, Gallimard, coll. « Encyclopédie de la Pléiade », 1961, p. 1247-1366.

Les Problèmes posés par l'édition critique des textes anciens et médiévaux, dir. Jacqueline HAMESSE, Louvain-la-Neuve, Institut d'études médiévales de l'Université catholique de Louvain, coll. « Textes, études, congrès », 1992.

Le Statut du scripteur au Moyen Âge, Actes du XII ${ }^{\mathrm{e}}$ Colloque scientifique du comité international de paléographie latine (Cluny, 17-20 juillet 1998), dir. Marie-Clotilde Hubert, Emmanuel Poulle, Marc H. SMith, Paris, École des Chartes, 2000.

Quentin, Dom Henri, Mémoire sur l'établissement du texte de la Vulgate, Rome, Desclée, 1922.

—, Essais de critique textuelle (ecdotique), Paris, A. Picard, 1926.

Stout, Selatie Edgar, «The Mind of the Scribe », The Classical Journal [en ligne], t. 22, 1927, p. 405-417, URL : http://www.jstor.org/stable/3288517 (page consultée le 14 décembre 2010).

Studies in stemmatology, dir. Pieter van REENEN, Margot van MulKen et Janet DYK, Amsterdam/Philadelphia, J. Benjamins, 1996. 
Studies in stemmatology. II, dir. Pieter van ReEnen, Aurelius Augustinus den Hollander, Margot van MulKen et Annelies Roeleveld, Amsterdam/New York, J. Benjamins, 2004.

Texts and transmission : a survey of the Latin classics, dir. Leighton Durham REYNOLDS, Oxford, Clarendon press, 1983.

VARVAro, Alberto, «Critica dei testi classica e romanza, problemi comuni ed esperienze diverse », Rendiconti dell'Accademia di Archeologia, Lettere e Belle Arti, 45, 1970, p. 73-117.

\section{L'attribution des textes et la stylométrie}

Contini, Gianfranco, Il Fiore e il Detto d'amore attribuibili a Dante Alighieri, Milano, Arnoldo Mondadori, coll. « Le opere di Dante Alighieri », 1985.

Delcourt, Christian, "Stylometry », Revue belge de philologie et d'histoire, 80, 2002, p. 979-1002.

Koppel, Moshe, Schler, Jonathan et Argamon, Shlomo, «Computational methods in authorship attribution », Journal of the American Society for Information Science \& Technology, 60, 2009, p. 9-26.

RUDMAN, Joseph, «The State of non-traditional authorship attribution studies - 2012 : some problems and solutions ", English Studies, t. 93, 2012, 3, p. 259-274, URL : http://www.tandfonline.com/doi/abs/10.1080/0013838X.2012.668785, page consultée le 30 janvier 2013.

« Stylometry and Authorship Attribution » (numéro spécial), dir. Javier CALLE-MARTín et Antonio MirandA-GarcíA, English studies, 93, 3, 2012, URL : http://www.tand fonline.com/doi/abs/10.1080/0013838X.2012.668788, page consultée le 30 janvier 2013.

\section{La copie et la part de l'auteur : questionnement du statut d'auteur, stratégies d'authentification.}

À plus d'un titre. Les titres des cuvres dans la littérature française du Moyen Âge au XX ${ }^{e}$ siècle, dir. Claude LACHET, Lyon, CEDIC, 2000.

L'Artiste et le commanditaire aux derniers siècles du Moyen Âge (XIII ${ }^{e}-X V I^{e}$ siècles), dir. Fabienne Joubert, Paris, Presses de l’Université de Paris-Sorbonne, 2001.

L'Auteur, éd. Alain BrunN, Paris, Flammarion, coll. « Corpus », 2001.

L'Autorité du passé dans les sociétés médiévales, dir. Jean-Marie SANSTERRE, Roma, École française de Rome, 2004, et plus particulièrement Olivier GUYOTJEANNIN, " Antiqua et authentica praedecessorum nostrorum nos ammonent: appel et rejet du passé chez les rédacteurs d'actes occidentaux (VIII-XIV siècle) », p. 9-25.

L'Autorité en littérature, dir. Emmanuel Bousu, Rennes, Presses Universitaires de Rennes, coll. « Interférences », 2010.

Author, Reader, Book: Medieval Authorship in Theory and Practice, dir. Stephen PARTRIDGE et Erik KWAKKEL, Toronto, University of Toronto Press, 2012. 
BADEL, Pierre-Yves, « Rhétorique et polémique dans les prologues de romans au Moyen Âge », Littérature, $\mathrm{n}^{\circ}$ 20, 1975, p. 81-94.

BARTHES, Roland, «La mort de l'auteur », Manteia, $\mathrm{n}^{0}$ 5, 1968, p. 12-17, rééd. dans Euvres complètes, en 3 vol. : vol. 1, Paris, Seuil, 1993, p. 491-495.

BAumgartner, Emmanuèle, "Masques de l'écrivain et masques de l'écriture dans les proses du Graal», dans Masques et déguisements dans la littérature médiévale, dir. Marie-Louise OlLIER, Montréal/Paris, Presses de 1'Université de Montréal/Vrin, coll. « Études médiévales », 1988, p. 167-175.

-, « Sur quelques constantes et variations de l'image de l'écrivain (XII - XIII ${ }^{\mathrm{e}}$ siècle) », dans Auctor et auctoritas, Invention et conformisme dans l'écriture médiévale, dir. Michel ZiMMERMANN, coll. « Mémoires et documents de l'École des chartes », 2001.

Berthelot, Anne, Figures et fonction de l'écrivain au XIII siècle, Montréal/Paris, Vrin, 1991.

Biaggini, Olivier, L'Auctoritas en Castille à la fin du XIII siècle. L'exemple de Gonzalo de Berceo, thèse dirigée par Michel GarCIA, Paris III, 1999.

Borges, Jorge Luis, «Pierre Ménard, auteur du Quichotte », Fictions, Paris, Gallimard, coll. « Folio », 1951.

Bouchet, Florence, Le Discours sur la lecture en France aux XIV et XV siècles: pratiques, poétique, imaginaire, Paris, Champion, 2008.

Brown, Cynthia, Poets, Patrons and Printers. Crisis of authority in late medieval France, Ithaca/London, Cornell University Press, 1995.

BRUNS, Gerald L., "The Originality of texts in a manuscript culture », Comparative Literature, vol. 32, $\mathrm{n}^{\mathrm{o}} 2$, 1980, p. 113-129.

Le Choix de la prose (XIII ${ }^{e} X V^{e}$ siècles), dir. Emmanuèle BAUMGARTNER, Cahiers de Recherches médiévales et humanistes, $\mathrm{n}^{\circ} 5,1998$, mis en ligne le 30 octobre 2000, URL : http://crm.revues.org/1292 (page consultée le 19 octobre 2014).

COMPagnon, Antoine, Qu'est-ce qu'un auteur? Cours de théorie de la littérature, URL : http://www.fabula.org/compagnon/auteur.php (page consultée le 19 octobre 2014).

DelsauX Olivier, "Les désignations des manuscrits originaux en moyen français », Original et originalité. Aspects historiques, philologiques et littéraires, actes $d u I V^{e}$ colloque de l'Association Internationale pour l'Étude du Moyen Français, Louvain-laNeuve, 20, 21 et 22 mai 2010, dir. Olivier DelsauX et Hélène Haug, Louvain-laNeuve, Presses universitaires de Louvain, 2011, p. 53-67.

Dictionnaire raisonné de l'Occident médiéval, dir. Jacques LE GofF et Jean-Claude SCHMITT, Paris, Fayard, 1999.

DOUDET, Estelle, «La condicion de l'hystoriographe : enquête sur une figure et un statut dans l'œuvre de George Chastelain », Le Moyen Âge, n ${ }^{\circ}$ CXII, 3-4, 2006, p. 545-556.

Foucault, Michel, «Qu'est-ce qu'un auteur?» (1969), Dits et Écrits, Paris, Gallimard, coll. « Bibliothèque des sciences humaines », 1994, t. I.

Le Goût du lecteur à la fin du Moyen Âge, dir. Danielle BoHLER, Paris, Le Léopard d'or, coll. « Cahiers du Léopard d'or », 2006.

GuENÉE, Bernard, Histoire et culture historique dans l'Occident médiéval, Paris, Aubier, 1980, p. 129-147. 
-, «L'historien et la compilation au XIII ${ }^{\mathrm{e}}$ siècle », Journal des savants, $\mathrm{n}^{\mathrm{0}}$ 1-3, 1985, p. 119-135.

Hasenohr, Geneviève, et Charon-Parent, Annie, «Le livre de part et d'autre de Gutenberg. Le livre manuscrit; D'un monde à l'autre », dans Histoire de la France littéraire. Naissances, Renaissances. Moyen Âge-XVI siècle, dir. Frank Lestringant et Michel ZINK, Paris, PUF, coll. Quadrige, 2006, p. 151-183.

Les Manuscrits autographes en français au Moyen Âge. Guide de recherche, dir. Olivier Delsaux et Tania VAn Hemelryck, Turnhout, Brepols, coll. «Texte, Codex et Contexte », 2014.

Marchello-Nizia, Christiane, «L'historien et son prologue : forme littéraire et stratégies discursives ", dans La Chronique et l'histoire au Moyen Âge, dir. Daniel PoIRION, Paris, Presses de l'Université de Paris-Sorbonne, 1986, p. 13-25.

MARICHAL, Robert, "Livre manuscrit», dans Dictionnaire des lettres françaises. Le Moyen Âge, dir. Robert Bossuat, Louis Pichard, Guy Raynaud De Lage, Fayard, 1964, rééd. 1994 sous la direction de Geneviève HASENOHR et Michel ZINK, coll. « Le Livre de poche », p. 941-949.

The Medieval Author in Medieval French Literature, dir. Virginie GREENE, New YorkBasingstoke, Palgrave Macmillan, coll. «Studies in Arthurian and Courtly Cultures », 2006.

La méthode critique au Moyen Âge, dir. Mireille CHAZAN et Gilbert DAHAN, Turnhout, Brepols, coll. « Bibliothèque d'histoire culturelle du Moyen Âge », 2006.

Minnis, Alastair J., Medieval Theory of Authorship: Scholastic literary attitudes in the later Middle Ages, London, Scolar Press, 1984.

Minnis, Alastair J., et ScotT, Alexander Brian, Medieval Literary Theory and Criticism c. 1100-c. 1375 : the Commentary-Tradition, Oxford, Clarendon Press, 1988.

La Mise en recueil des textes médiévaux, dir. Xavier Leroux, Babel, n 16, 2007.

Mouvances et jointures. Du manuscrit au texte médiéval. Actes du Colloque international organisé par le CeReS - Université de Limoges, 21-23 novembre 2002, dir. Milena Mikhailova, Orléans, Éditions Paradigme, coll. « Medievalia », 2005.

Performing Medieval Narrative, dir. Evelyn Birge Vitz, Nancy Freeman Regalado et Marilyn LAWrence, Cambridge/Rochester, D. S. Brewer, 2005, partie II : « Medieval Performance and the Book ».

Postures d'auteurs : du Moyen Âge à la modernité, dir. Jérôme MeIzoz, Jean-Claude MüHLETHALER et Delphine Burghgraeve, en ligne, URL : http://www.fabula.org/ colloques/sommaire2341.php (page consultée le 18 octobre 2014).

La Question de l'auteur, Actes du XXX Congrès de la Société des hispanistes français, Brest, 18-19-20 mai 2002, dir. Corinne et Manuel MonTOYA, Brest, Université de Bretagne Occidentale, 2002, p. 131-146.

RENo, Christine, «Les manuscrits originaux de la Cité des dames de Christine de Pizan », dans L'Écrit et le manuscrit à la fin du Moyen Âge, dir. Tania VAN HEMELRYCK et Céline VAn Hoorebeeck, Turnhout, Brepols, coll. « Texte, Codex et Contexte », 2006, p. 267-276.

Samoyaut, Tiphaine, L'Intertextualité. Mémoire de la Littérature, Paris, Armand Colin, 2005. 
Seuils de l'ouvre dans le texte médiéval, dir. Emmanuèle BAUMGARTNER et Laurence HARF-LANCNER, Paris, Presses Sorbonne Nouvelle, vol. 1 et 2, 2002.

SUARD, François, «Le passage à la prose », Cahiers de recherches médiévales, $\mathrm{n}^{0}$ 12, La tradition épique, du Moyen Âge au XIX siècle, dir. François Suard, 2005, p. 29-43, mis en ligne le 30 décembre 2008, URL : http://crm.revues.org/2182 (page consultée le 15 juillet 2014).

-, François, Guillaume d'Orange, étude du roman en prose, Paris, Champion, coll. « Bibliothèque du XV $\mathrm{XV}^{\mathrm{e}}$ siècle », 1979.

Le Turpin français, dit le Turpin I, éd. Ronald Noel WALPOLE, Toronto/Buffalo/London, University of Toronto Press, coll. « Toronto Medieval Texts and Translations », 1985.

TRACHSLER, Richard, «Les auteurs des romans arthuriens en prose. De l'anonyme au pseudonyme », conférence au Collège de France du 9 janvier 2013, mise en ligne le $1^{\text {er }}$ septembre 2013, URL : http://www.college-de-france.fr/site/michel-zink/seminar-201301-09-11h30.htm (page consultée le 19 octobre 2014).

VAN HEMELRYCK, Tania, " Of books and other miscellaneous revolutions. Medieval miscellanies in context », dans Collections in context. The Organization of knowledge and community in Europe, dir. Karen Fresco and Anne D. Hedeman, Columbus, The Ohio State University Press, 2011, p. 288-293.

VINCENSINI, Jean-Jacques, « Comprendre, décrire, interpréter un motif narratif. L'exemple de la libération d'une femme immergée dans l'eau par un jaloux », dans Dans l'eau, sous l'eau: le monde aquatique au Moyen Âge, dir. Danièle JAMES-RAOUL et Claude Thomasset, Paris, Presses de l'Université de Paris-Sorbonne, 2002, p. 387-411.

Zumthor, Paul, Essai de poétique médiévale, Paris, Éditions du Seuil, coll. « Poétique », 1972, rééd. coll. « Points Essais », 2000.

ZuMTHOR, Paul, « L'oubli et la tradition », Le Genre humain, nº 18, 1988, p. 105-117. 